\title{
Resistência e rigidez da madeira de espécies florestais submetidas a campo de apodrecimento
}

\author{
Douglas Edson Carvalho ${ }^{1 *}$ Márcio Pereira Da Rocha ${ }^{1} \quad$ Ricardo Jorge Klitzke $^{1}$ Pedro Henrique Gonzalez De \\ Cademartori $^{1}$ Claudio Gumane Francisco Juizo ${ }^{1}$ Ana Paula Marques Martins ${ }^{1}$
}

${ }^{1}$ Universidade Federal Do Paraná, Av. Prefeito Lothário Meissner, 632 - Jardim Botânico, Curitiba - PR, 80210-170

*Author for correspondence: douglasedsoncarvalho@gmail.com

Received: September 2018/ Accepted: February 2019 / Published: March 2019

\begin{abstract}
Resumo
Ensaio de campo de apodrecimento por períodos determinados configura-se como o método mais relevante para avaliar as propriedades mecânicas da madeira. Diante disso a pesquisa teve como objetivo avaliar a resistência e rigidez das madeiras de Eucalyptus dunnii, Eucalyptus robusta, Eucalyptus tereticornis e Hovenia dulcis submetidas à exposição de agentes bióticos e abióticos em ambientes de campo aberto e floresta nativa. Foram confeccionados 240 corpos-de-prova com dimensões de 20 x 20 x $300 \mathrm{~mm}$ (espessura x largura x comprimento), respectivamente. As madeiras permaneceram expostas por um período de 405 dias, sendo avaliados a cada 45 dias em ensaios de flexão estática, para determinação do módulo de elasticidade (MOE) e módulo de ruptura (MOR). A madeira de Eucalyptus tereticornis apresentou valores médios superiores às demais espécies, sendo 16469,2; 14700,8 e $11025,2 \mathrm{MPa}$ para MOE e 132,71; 81,57 e 69,93 MPa para MOR, correspondendo à testemunha, ambiente de campo e ambiente de floresta, respectivamente. A madeira de Eucalyptus dunnii apresenta maior queda na resistência a flexão estática do material natural em relação ao material exposto nos ambientes. Eucalyptus robusta não apresentou alteração significativa de resistência e rigidez entre os corpos-de-prova testemunhas e os expostos aos ambientes. Eucalyptus tereticornis apresenta MOE e MOR após exposição superior as demais espécies. As madeiras expostas em ambiente de campo apresentaram redução de MOE e MOR em comparação com as madeiras expostas no ambiente de floresta para todas as espécies.

Palavras chave: Biodeterioração, módulo de elasticidade; módulo de ruptura; flexão estática; Eucalyptus
\end{abstract}

\footnotetext{
Abstract

Field trial of rotting for certain periods is the most relevant method to evaluate mechanical properties of wood. With this, the objective of this research was to evaluate the strength and stiffness of the wood of Eucalyptus dunnii, Eucalyptus robusta, Eucalyptus tereticornis and Hovenia dulcis woods, exposed to biotic and abiotic agents attack, in open-field and inside forest. Two-hundred forty samples with dimensions of $20 \times 20 \times 300 \mathrm{~mm}$ (thickness $\mathrm{x}$ width $\mathrm{x}$ length) were prepared. The wood were exposed for 405 days, being collected every 45 days for bending strength tests to determine the modulus of elasticity (MOE) and modulus of rupture (MOR). Eucalyptus tereticornis wood presented the average values superior to the other species, being 16469.2 ; 14700.8 and $11025.2 \mathrm{MPa}$ for MOE and $132.71 ; 81.57$ and $69.93 \mathrm{MPa}$ for MOR, corresponding to the control, field environment and forest environment respectively. The wood of Eucalyptus dunnii shows a greater decrease in the resistance to static bending of the natural material in relation to the material exposed in the environments. Eucalyptus robust didn't present significant
}

alteration of resistance and stiffness between the test specimens and those exposed to the environments. Eucalyptus tereticornis presents MOE and MOR after superior exposure to the other species. The wood exposed in the open-field resulted in reduction of MOE and MOR compared to the woods exposed inside the forest for all species.

Key words: Biodeterioration; modulus of elasticity; modulus of rupture; bending strength; Eucalyptus.

\section{Introdução}

É de conhecimento universal que a madeira é utilizada pela humanidade desde o início da civilização como um dos principais materiais para cobrir necessidades construtivas, de utensílios diários, bem como para diversas expressões estéticas e artísticas. Porém, apesar do seu uso intensivo, a madeira quando está em serviço enfrenta algumas ameaças provenientes do meio ambiente: umidade, radiação UV e fungos (Makarona et al. 2017), além de organismos outros xilófagos.

Estes organismos e agentes, quando atacam e incidem sobre madeira, podem afetar a sua estrutura, reduzindo a sua durabilidade natural e as propriedades mecânicas. Porém, o grau de deterioração de cada madeira ocorre em decorrência da sua resistência, ou seja, algumas espécies são mais suscetíveis ao desgaste em relação às outras, sendo por isso necessário o conhecimento dos esforços mínimos e máximos os quais as espécies madeireiras admitem, quando expostas em ambientes adversos e principalmente em contato com o solo.

Segundo Mattos et al. (2014), a taxa de deterioração de um material biodegradável depende diretamente do ambiente exato ao qual está exposto, dentre esses materiais biodegradáveis, a madeira é considerada durável em relação à capacidade de manter suas propriedades estruturais sob a ação do intemperismo, embora os ataques de xilófagos devam ser levados em consideração.

De acordo com Stangerlin et al. (2015), a avaliação da durabilidade natural de madeiras pode ser realizada por inspeção visual da sanidade da peça em uso. Porém, para avaliar a resistência à deterioração da madeira a análise da perda de propriedades físicas e mecânicas configura-se como parâmetro essencial (Simsek et al. 2010; Ali et al, 2011 ). Todavia, para Trevisan et al. (2007), a caracterização mecânica certamente fornece respostas complementares aos processos que envolvem a deterioração da madeira, informações estas que visam à melhor compreensão do comportamento da espécie. Assim permite o correto aproveitamento de cada característica resultando na redução de gastos e desperdícios por reposição de peças, especialmente quando estas estão em contato com fontes que possam causar uma deterioração em curto espaço de tempo, reduzindo a vida útil do material. 
Segundo Stangerlin et al. (2010), dentre os ensaios para obtenção de esforços máximos e mínimos que a madeira permite, pode-se destacar a flexão estática, para observar o comportamento das peças submetidas a esforços de tração, compressão e cisalhamento por meio do flexionamento causado por uma carga aplicada na face tangencial com vista à sua deformação. $\mathrm{O}$ comportamento e esforços máximos e mínimos da peça são obtidos pelos valores de módulos de elasticidade (MOE) e de ruptura (MOR), sendo que estes parâmetros também variam de acordo com a espécie, umidade da madeira, massa específica e tempo de duração da carga durante o ensaio mecânico, entre outros fatores (Braz et al. 2013).

Nesse contexto, é importante ensaiar principalmente madeiras que serão empregadas em contato direto com solo, tais como postes e mourões. Shimizu e Carvalho (2000) apontam um dos requisitos mais importantes para madeiras usadas como mourões e postes é a durabilidade natural da madeira, pois essas tem contato direto com o solo. Nesse aspecto, dentre as espécies de eucalipto mais indicadas estão o Eucalyptus robusta e o Eucalyptus tereticornis. Todavia, testar outras madeiras tem papel fundamental, a fim de aumentar a gama de espécies para os mais diversos usos. A madeira de Eucalyptus dunnii e de Hovenia dulcis apresentam potencial especial para produção de madeira serrada, porém, dado o rápido crescimento, a qualidade do fuste e a vasta utilização dessas espécie na região Sul do Brasil, é importante conhecer a sua durabilidade natural para que estas espécies possam ser uma opção também na utilização em contato direto com o solo, como mourões e postes (Shimizu e Carvalho 2000; Carvalho 1994).

Portanto, é de suma importância a realização de estudos que busquem identificar madeiras com maior resistência e rigidez quando submetidas a condições adversas de uso. Assim sendo, a realização de experimentos com campos de apodrecimento, tem sido uma alternativa, pois os mesmos buscam reproduzir as condições reais a que as peças de madeiras possam estar sujeitas. Melo et al. (2010), Mattos et al. (2013) e Cookson et al. (2014) salientam que os ensaios com madeiras em campo de apodrecimento permitem obter resultados confiáveis e mais fidedignos quanto à durabilidade, uma vez que no campo a madeira fica exposta a condições mais severas de deterioração, principalmente quando em contato com o solo. Deste modo, o estudo da resistência de espécies florestais em ambientes adversos visa elucidar o comportamento das madeiras frente a estas situações, especialmente de espécies exóticas plantadas consideradas de rápido crescimento.

Diante do exposto o estudo foi desenvolvido para avaliar se a resistência à flexão estática de madeiras de florestas plantadas é afetada quando submetidas a campo de apodrecimento, em ambientes de campo e de floresta em função do tempo de exposição.

\section{Material e Métodos}

No presente estudo foram utilizadas madeiras de Eucalyptus dunnii Maiden, Eucalyptus robusta Sm, Eucalyptus tereticornis Sm e Hovenia dulcis Thunberg, todas com 35 anos de idade, provenientes do Centro de Pesquisa em Recursos Florestais da Fundação Estadual de Pesquisa Agropecuária (Fepagro Florestas), localizada no distrito de Boca do Monte, município de Santa Maria, Rio Grande do Sul. Após a derrubada de três árvores por espécie, foram extraídas pranchas com 3,0 metros de comprimento da primeira tora de cada espécie as quais foram utilizadas para preparo dos corpos-de-prova.

Campo de apodrecimento e preparo de corpos-de-prova
O campo de apodrecimento foi estabelecido em dois ambientes: campo aberto sem cobertura vegetal, nas coordenadas $29^{\circ} 43^{\prime} 12.2^{\prime \prime} \mathrm{S}$ e $53^{\circ} 43^{\prime} 12.3^{\prime \prime} \mathrm{W}$; ambiente de floresta, composto por espécies nativas, nas coordenadas $29^{\circ} 43^{\prime} 14.1^{\prime \prime S}$ e $53^{\circ} 43^{\prime} 13.7^{\prime \prime} \mathrm{W}$. Foi realizada coleta da camada de solo até $30 \mathrm{~cm}$ para caracterização do teor de matéria orgânica dos ambientes analisados. Os corpos-deprova foram distribuídos aleatoriamente a uma distância de $10 \mathrm{~cm}$ entre si e $30 \mathrm{~cm}$ entre linha em uma área aproximada de $6 \mathrm{~m}^{2}$ para cada ambiente. A temperatura média durante o tempo de estabelecimento do experimento variou de $14{ }^{\circ} \mathrm{C}$ (inverno) a $28{ }^{\circ} \mathrm{C}$ (verão) e a umidade relativa esteve na faixa de $69 \%$ a $79 \%$.

Foram confeccionados e selecionados 60 corpos-deprova por espécie totalizando 240 corpos-de-prova para as quatro espécies. As análises dos corpos de prova foram efetuadas em intervalos de 45 dias, coletando-se três amostras de cada espécie e de cada ambiente de exposição, perfazendo seis corpos-de-prova, totalizando 24 corpos-deprova das quatro espécies testadas em cada amostragem e seis amostras utilizadas como testemunhas. Os mesmos foram obtidos da madeira de cerne, livres de defeitos, tais como esmoados, rachaduras e nós, com dimensões de $20 \mathrm{x}$ 20 x $300 \mathrm{~mm}$ (largura x espessura x comprimento), conforme a norma COPANT 30:1 - 006 (1972).

Posteriormente, foram acondicionados em câmara climática na temperatura de $20 \pm 2{ }^{\circ} \mathrm{C}$ e umidade relativa de $65 \pm 5 \%$ até atingirem peso constante. Em seguida, foram levados a campo, e fixados no solo até metade do comprimento $(150 \mathrm{~mm})$, região de afloramento onde à presença de oxigênio e umidade que contribui para o crescimento dos organismos xilófagos, sendo a região mais sensível das amostras, expostas por um período de 405 dias.

\section{Avaliação do módulo de elasticidade e de ruptura à flexão estática}

A cada coleta os corpos-de-prova foram limpos para a remoção de partículas de solo e novamente acondicionados em câmara climática até obterem peso constante. Em seguida, os mesmos foram ensaiados em flexão estática, de acordo com os procedimentos da norma COPANT (1972), utilizando-se uma máquina universal de ensaios, com capacidade para 20 toneladas, aplicando uma carga contínua no corpo-de-prova, com velocidade de $1,0 \mathrm{~mm} /$ minuto, obtendo-se, assim, as informações necessárias para o cálculo dos módulos de elasticidade (MOE) e de ruptura (MOR).

\section{Análise estatística}

Previamente à análise de variância (ANOVA) foram realizados testes para satisfazer os pressupostos de normalidade dos dados, homogeneidade das variâncias e independência dos resíduos. Para análise dos resultados procedeu-se com ANOVA $(\mathrm{p} \leq 0,05)$ para MOE e MOR, pelo delineamento inteiramente casualizado em arranjo bifatorial, sendo "espécie" o fator 1 com 4 níveis e "ambiente de exposição" o fator 2 com 2 níveis. Os fatores e as interações que apresentaram diferença significativa pela ANOVA tiveram as suas médias comparadas pelo teste de Tukey $(\mathrm{p} \leq 0,05)$. Complementarmente, foram elaborados gráficos da variação da resistência e rigidez durante o período de exposição dos corpos-de-prova nos dois ambientes para cada espécie avaliada.

\section{Resultados e discussão}

As peças do ambiente de campo aberto apresentaram MOE superior e diferente estatisticamente das peças expostas no ambiente de floresta, com exceção para a madeira de E. robusta, a qual não apresentou diferença estatística entre testemunha e ambientes de exposição tanto 
para MOE como para MOR, indicando que esta espécie apresenta resistência quando exposta a ambientes adversos.

Carvalho et al. (2015) estudando a durabilidade natural de espécies florestais em laboratório verificaram que a espécie E. robusta, apesar não ter apresentado a massa específica aparente mais elevada dentre as espécies estudadas, apresentou a maior resistência à ataque de fungos o que favorece as propriedades mecânicas da espécie quando exposta à diversos ambientes. Essa maior resistência à organismos xilófagos pode ser atribuída à maior concentração de extrativos na madeira dessa espécie. Em

Tabela 1. Valores médios de módulo de elasticidade e módulo de ruptura observados para as espécies Eucalyptus dunnii, Eucalyptus robusta, Eucalyptus tereticornis e Hovenia dulcis em amostras testemunha e expostas em ambientes de campo e de floresta após 405 dias.

\begin{tabular}{|c|c|c|c|c|c|}
\hline \multirow{2}{*}{ Variável } & \multirow{2}{*}{ Espécie } & \multicolumn{3}{|c|}{ Ambiente de exposição } & \multirow{2}{*}{ C.V. $(\%)$} \\
\hline & & Testemunha & Campo & Floresta & \\
\hline \multirow{4}{*}{ MOE (MPa) } & E. tereticornis & $16469,2 \mathrm{~A} \mathrm{a}$ & $14700,8 \mathrm{~A} \mathrm{a}$ & $11025,2 \mathrm{~B} \mathrm{a}$ & \multirow{4}{*}{26,4} \\
\hline & H. dulcis & $11137,6 \mathrm{~A} \mathrm{bc}$ & $9762,4 \mathrm{AB} b c$ & $8885,1 \mathrm{~B} \mathrm{~b}$ & \\
\hline & E. robusta & $7892,6 \mathrm{~A} \mathrm{c}$ & $8263,5 \mathrm{~A} \mathrm{c}$ & 7996,9 A b & \\
\hline & E. dunnii & $12237,4 \mathrm{~A} \mathrm{~b}$ & $10779,7 \mathrm{~A} \mathrm{~b}$ & $6972,8 \mathrm{~B} \mathrm{~b}$ & \\
\hline \multirow{4}{*}{ MOR (MPa) } & E. tereticornis & $132,71 \mathrm{~A} \mathrm{a}$ & $81,57 \mathrm{~B}$ a & $69,93 \mathrm{~B} \mathrm{a}$ & \multirow{4}{*}{29,9} \\
\hline & H. dulcis & $113,80 \mathrm{~A} \mathrm{a}$ & $81,28 \mathrm{~B} \mathrm{a}$ & $69,49 \mathrm{~B} \mathrm{a}$ & \\
\hline & E. robusta & $72,16 \mathrm{~A} \mathrm{~b}$ & $64,26 \mathrm{~A} \mathrm{~b}$ & $62,62 \mathrm{~A} \mathrm{a}$ & \\
\hline & E. dunnii & $116,97 \mathrm{~A} \mathrm{a}$ & $66,33 \mathrm{~B} \mathrm{ab}$ & $40,72 \mathrm{C} \mathrm{b}$ & \\
\hline
\end{tabular}
CV\%: Coeficiente de Variação Experimental.

Houve diferença significativa para o MOE entre as amostras testemunhas e expostas no ambiente de floresta, com reduções percentuais de $20 \%, 33 \%$ e $43 \%$ para as espécies H. dulcis, E. tereticornis e E. dunnii, respectivamente. Comportamento semelhante foi percebido para o MOR nas mesmas espécies, em que as reduções percentuais foram de $39 \%$, $47 \%$ e $65 \%$, respectivamente (Figura 1). Estatisticamente não se observa diferença entre as testemunhas e as peças do ambiente de campo para o MOE (Tabela 1). Porém para o MOR verificou-se diferença significativa, com reduções percentuais de $28 \%$, $38 \%$ e $43 \%$ para as espécies $H$. dulcis, E. tereticornis e E. dunnii, respectivamente (Figura 1).
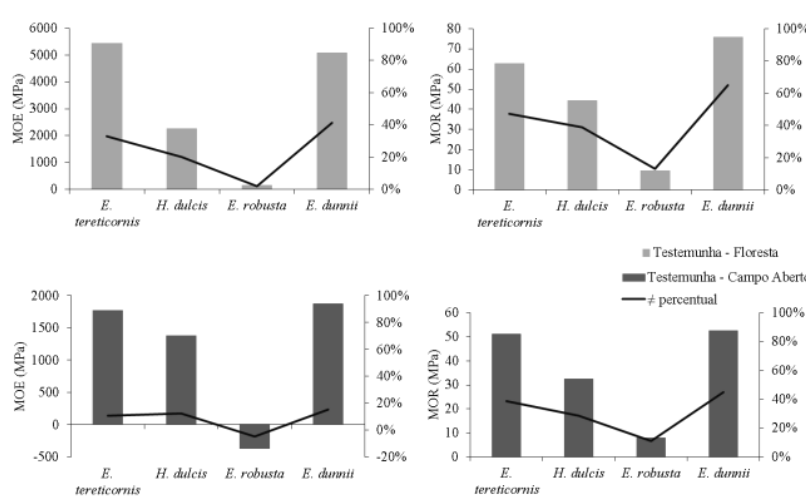

Figura 1. Comportamento da diferença percentual média de MOE e MOR para diferentes espécies submetidas a campo de apodrecimento em campo aberto e floresta em relação à testemunha.

No ambiente de campo, E. robusta apresentou MOE inferior em relação às demais espécies, porém sem diferença significativa com $H$. dulcis, contrariamente às peças de $E$. tereticornis que apresentaram resultados superiores com diferenças estatísticas entre as demais. Por sua vez, no ambiente de floresta, E. dunnii apresentou MOE inferior, todavia não diferiu estatisticamente de $H$. dulcis e $E$. robusta, sendo mais uma vez E. tereticornis a espécie com estudo realizado por Pereira (2003), o qual avaliou as características de seis espécies de eucalipto, apontou a espécie E. robusta com maior concentração de extrativos, confirmando tal afirmação.

Verificamos que a interação dos fatores "ambiente de exposição" e "espécie" foi significativa a $5 \%$ de probabilidade de erro e com isso foi desdobrada a interação entre os fatores (Tabela 1) em que se observa que a exposição das madeiras nos ambientes de campo e de floresta ocasionou alterações do MOE. 


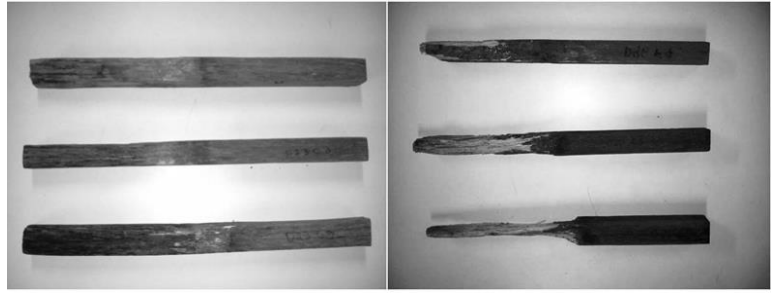

Figura 2. Corpos-de-prova de Eucalyptus dunnii após retirada do ambiente de campo (A) e ambiente de floresta (B).

\section{Variação do MOR e MOE}

Notamos que E. dunnii apresentou as maiores oscilações entre os ambientes para o MOE e MOR (Figuras 3 e 4), assim como em relação aos corpos-de-prova das testemunhas. Para o MOR, a espécie E. dunnii apresentou as maiores oscilações, nas madeiras expostas nos dois ambientes em relação às demais espécies. No entanto, foi possível observar que tanto em ambiente de campo, assim como em ambiente de floresta, houve um decréscimo da resistência ao longo do tempo de exposição para todas as espécies.

E. dunnii se destaca por apresentar maior percentual de redução resistência, resultando na perda de material avaliado após 360 dias de exposição, fato que pode estar associado à volatização de compostos químicos moleculares durante o período de exposição, aliado ao consumo dos mesmos devido à preferência alimentar de organismos atuantes no local, os quais podem ter gerado uma perda superior de compostos responsáveis pela resistência mecânica da madeira, consequentemente a espécie não é indicada para usos externos em contato com o solo. Legitimando tal informação, Mattos et al. (2013), descrevem que esta situação acontece porque a madeira, quando exposta em diferentes estágios de deterioração, apresenta redução da compactação do material celulósico na parede celular, proporcionando perda de massa e da elasticidade da mesma.
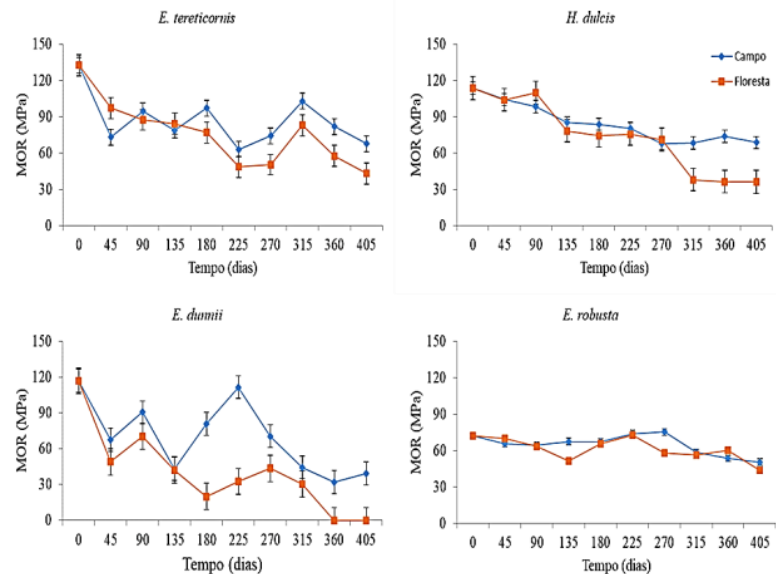

Figura 3. Módulo de ruptura (MOR) no decorrer do tempo em ambiente de campo e floresta para as quatro espécies. As barras nas colunas indicam o desvio padrão.

Panshin \& De Zeeuw (1980) alegaram que madeiras mais densas ou menos porosas oferecem maior resistência ao apodrecimento por fungos, no entanto, essa situação não foi observada por Carvalho et al. (2016), onde os autores avaliaram as mesmas amostras e espécies utilizadas neste estudo, obtendo a massa específica aparente a $12 \%$ de 0,67 $\mathrm{g} / \mathrm{cm}^{3}$ para $H$. dulcis, $0,75 \mathrm{~g} / \mathrm{cm}^{3}$ para E. robusta, $0,90 \mathrm{~g} / \mathrm{cm}^{3}$ para E. tereticornis e $0,88 \mathrm{~g} / \mathrm{cm}^{3}$ para $E$. dunnii, a qual teve maiores perdas de resistência.
Apesar da massa específica elevada da espécie E. dunnii, esta apresentou redução superior da resistência e rigidez em comparação com as demais espécies. Com esta informação infere-se que não só a massa específica tem influência sobre a durabilidade e resistência da madeira, mas que os teores de extrativos e seus graus de toxidez, assim como os constituintes de alto e baixo peso molecular apresentam relação direta com tais propriedades, conforme Oliveira et al. (2005) e Stangerlin et al. (2013).

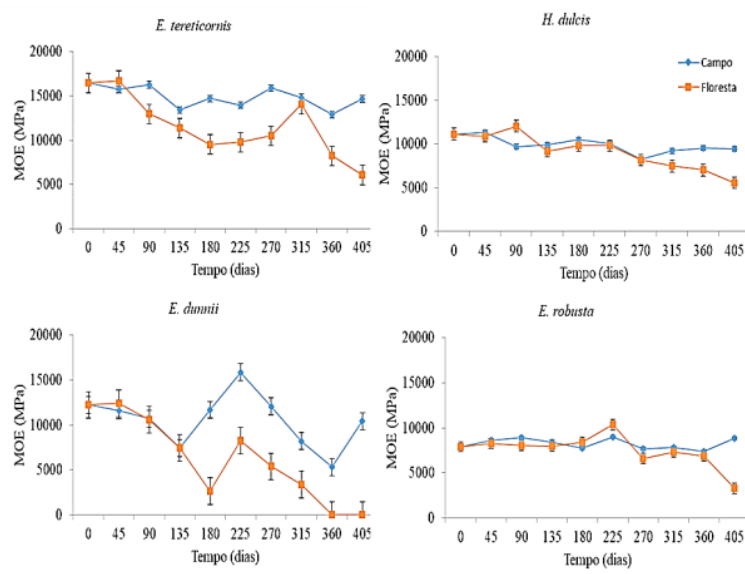

Figura 4. Módulo de Elasticidade (MOE) no decorrer do tempo em ambiente de campo e floresta para diferentes espécies. As barras nas colunas indicam o desvio padrão.

Da mesma forma, a espécie E. dunnii apresentou as maiores diferenças e oscilações entre campo e floresta para o MOE, durante o estudo. Ao final do período analisado, $E$. tereticornis apresentou os valores médios superiores em relação às demais espécies para o $\mathrm{MOE}$, em ambos os ambientes, com algumas oscilações ao longo do tempo. Entretanto, a espécie que indicou comportamento mais homogêneo no MOE foi E. robusta, não apresentando grandes variações entre os ambientes (Figura 4) e em relação a testemunha (Tabela 1), contrastando com o comportamento das demais espécies.

Este fato é preponderante para a escolha da espécie e aplicação da madeira em situações desfavoráveis de uso, como quando em contato com o solo onde há riscos e problemas associados a redução de resistência e rigidez mecânica. Pois, segundo Ali et al., (2011), o período que a madeira fica exposta deixa a mesma suscetível à ação de organismos como cupins e fungos, os quais degradam a parede celular e consequentemente reduz as propriedades mecânicas.

O E. tereticornis apresentou acréscimo de MOR para o ambiente de campo e floresta e de MOE para o ambiente de floresta entre 270 e 315 dias e redução após este período (Figura 4). Este fato está relacionado à precipitação em tal período, inicialmente com aumento e posterior diminuição (Figura 5). Esta situação também pode ser associada ao comportamento observado para $E$. dunnii o qual começou a ser evidenciado à partir de 135 dias, onde ocorre uma redução da precipitação até 180 dias, indicando que esta espécie é mais suscetível a alterações quando exposta a ambiente com umidade. Isto está relacionado com a redução das ligações de hidrogênio entre os polímeros da parede celular da madeira, o que reduz a resistência mecânica da madeira (Rowell, 2012). 


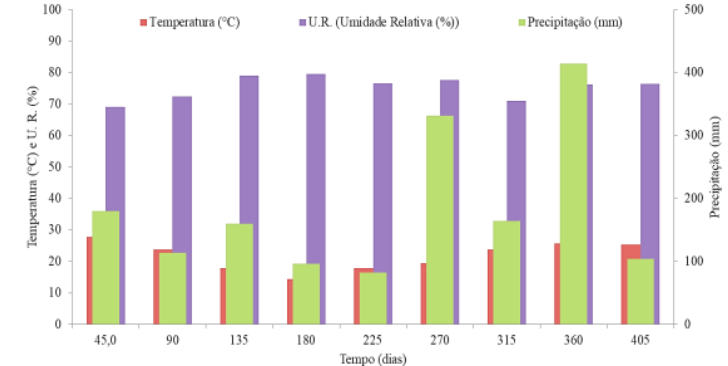

Figura 5. Comportamento das variáveis climáticas no período do estudo.

A natureza higroscópica da madeira gera uma relação íntima com o ambiente de uso da mesma, ocasionando absorção e perda de água, portanto a aumento e redução do nível pluviométrico do local de exposição ocasiona alterações anisotrópicas das peças acarretando em fissuras, que consequentemente reduzem a resistência e rigidez da madeira. O aumento observado em MOR e MOE ocorre primeiro no ambiente de campo, tanto para E. tereticornis quanto para $E$. dunnii, pois é onde não há proteção vegetal e a água oriunda de precipitações evapora em um espaço de tempo menor em relação ao ambiente de floresta, minimizando o efeito de microclima adequado para ação de organismos xilófagos.

Percebe-se que a perda da rigidez da madeira de todas as espécies foi influenciada pela condição de exposição ao intemperismo, sendo sempre maior em ambiente de floresta em comparação ao ambiente de campo devido as características dos solos e do microclima. Isto porque em ambiente de floresta foi observado maior teor de matéria orgânica no solo $(3,3 \%)$ em comparação ao solo de campo aberto $(1,9 \%)$ favorecendo, assim, a maior disponibilidade de microorganismos xilófagos que são capazes de consumir a lignina que é um dos principais constituintes químicos moleculares responsáveis pela rigidez da madeira.

\section{Conclusões}

E. dunnii apresenta maior queda na resistência a flexão estática do material natural em relação ao material exposto nos ambientes de campo e floresta, não sendo indicada pra uso em tais ambientes.

E. tereticornis apresenta resistência e rigidez final superior em comparação as demais espécies nos ambientes de campo aberto e floresta.

$E$. robusta não sofre influência do tempo e dos ambientes em sua resistência e rigidez, sendo indicada pra usos em contato com o solo.

As espécies estudadas apresentam uma tendência de redução da resistência à flexão estática (MOR e MOE) quando expostas ao ambiente de floresta em comparação com o ambiente de campo.

\section{Referências}

Ali AC, Uetimane Júnior E, Råberg U, Terziev N (2011) Comparative natural durability of five wood species from Mozambique. International Biodeterioration \& Biodegradation, 65 (11): 768-776. DOI: https://doi.org/10.1016/j.ibiod.2011.03.010

Braz RL, Oliveira JTS, Rodrigues BP, Arantes MDC (2013) Propriedades físicas e mecânicas da madeira de Toona ciliata em diferentes idades. Floresta, 43(4): 663 - 670. http://dx.doi.org/10.5380/rf.v43i4.30559

Comisión Panamericana de Normas Técnicas - COPANT 30: 1 - 006 (1972). Maderas: método de determinación de flexión estática.
Carvalho PER (1994) Ecologia, silvicultura e usos da uvado-japão (Hovenia dulcis Thunberg). Colombo: EMBRAPA-CNPFlorestas, 24p. (EMBRAPACNPFlorestas. Circular Técnica, 23).

Carvalho DE, Martins APM, Santini EJ, Freitas LS, Talgatti M, Susin F (2016) Natural durability of Eucalyptus dunnii Maiden, Eucalyptus robusta Sm., Eucalyptus tereticornis Sm. and Hovenia dulcis Thunb. wood in field and forest environment. Árvore, 40(2): 363-370. http://dx.doi.org/10.1590/0100-67622016000200019

Carvalho DE, Santini EJ, Gouveia FN, Rocha MP. Resistência natural de quatro espécies florestais submetidas a ensaio com fungos apodrecedores. Floresta e Ambiente, 22(2): 271-276

http://dx.doi.org/10.1590/2179-8087.105914

Cookson LJ, Page D, Singh T (2014) Accelerated aboveground decay testing in Australia and New Zealand. International Biodeterioration \& Biodegradation, 86: 210-217.

Pereira JCD. Características das madeiras de seis espécies de eucalipto plantadas em Colombo-PR. Colombo: Embrapa Florestas, 2003.

Makarona E, Koutzagioti C, Salmas C, Ntalos G, Skoulikidou MC, Tsamis C (2017) Enhancing wood resistance to humidity with nanostructured $\mathrm{ZnO}$ coatings. Nano-Structures \& Nano-Objects, 10: 57-68. DOI: https://doi.org/10.1016/j.nanoso.2017.03.003

Mattos Bd, Cademartori PHG, Lourençon TV; Gatto DA, Magalhães WLE (2014). Biodeterioration of wood from two fast-growing eucalypts exposed to field test. International Biodeterioration \& Biodegradation, 93: 210-215. https://doi.org/10.1016/j.ibiod.2014.04.027

Mattos BD, Gatto DA, Cademartori PHG, Stangerlin DM, Beltrame R (2013) Durabilidade a campo da madeira de três espécies de Eucalyptus tratadas por imersão simples. Revista Brasileira de Ciências Agrárias, 8(4): 648-655. DOI:10.5039/agraria.v8i4a3050

Melo RR, Stangerlin DM, Santini EJ, Haselein CR, Gatto DA, Susin F (2010) Durabilidade natural da madeira de três espécies florestais em ensaios de campo. Ciência Florestal, 20(2): 357-365.

Oliveira JTS, Hellmeister JC, Filho MT (2005) Variação do teor de umidade e da densidade básica na madeira de sete espécies de eucalipto. Árvore, 29(1): 115-127.

Panshin AJ, De Zeeuw C (1980) Textbook of wood technology. 4 ed. New York. 722p.

Ribeiro MA, Stangerlin DM, Souza AP de, Cardoso GV, Calegari L, Gatto DA (2014) Durabilidade natural da madeira de jequitibá em ensaios de deterioração em campo aberto e floresta durante as estações de seca e chuva. Comunicata Scientiae, 5(4): 402-411.

Rowell RM (2012) Handbook of Wood Chemistry and Wood Composites. 2 ed. CRC Press, London, New York, Singapore 703 p.

Stangerlin DM, Melo RR de, Gatto DA, Cademartori PHG de (2010) Propriedades de flexão estática da madeira de Carya illinoinensis em duas condições de umidade. Ciência da Madeira, 1(02): 70-79. 
Stangerlin DM, Costa AF, Garlet A, Pastore TCM (2013)

Resistência natural da madeira de três espécies amazônicas submetidas ao ataque de fungos apodrecedores. Ciência da Madeira, 4: 15-32.

Stangerlin DM, Corassa JN, Gatto DA, Pereira RL, Castelo PAR (2015) Caracterização mecânica de madeiras deterioradas em campo por meio de ultrassom e flexão estática. Comunicata Scientiae, 6(3): 365-372.

Silveira AG da, Trevisan R, Santini EJ, Cancian LC, Mariano LG (2016) Deterioração da madeira de acácianegra em dois ambientes de exposição. Scientia Agraria Paranaensis, 15(3) 251-255. DOI: http://dx.doi.org/10.18188/1983-1471/sap.v15n3p251255

Simsek H, Baysal E, Peker H (2010) Some mechanical properties and decay resistance of wood impregnated with environmentally-friendly borates. Construction and Building Materials, 24 (11): 2279-2284. DOI: https://doi.org/10.1016/j.conbuildmat.2010.04.028

Shimizu JY, Carvalho PER (2000) Primeira aproximação na indicação de eucaliptos para produção de madeira na região de Quaraí, RS. Boletim de Pesquisa Florestal, 40: 101-110.

Trevisan H, Tieppo FMM, Carvalho AG de, Lelis RCC (2007) Avaliação de propriedades físicas e mecânicas da madeira de cinco espécies florestais em função da deterioração em dois ambientes. Árvore, 31 (1): 30-37.

Vivin MA, Santini EJ, Modes KS, Carvalho DE, Morais WWC (2014) Resistência biológica da madeira tratada de duas espécies de Eucalyptus em ensaio de campo. Pesquisa Florestal Brasileira, 34(80): 425-433. DOI: 10.4336/2014.pfb.34.80.545 